\title{
THERMAL EXPANSION STUDY OF A UVAROVITE RICH GARNET
}

\author{
G. PARTHASARTHY and R. SRINIVASAN \\ National Geophysical Research Institute, Hyderabad - 50000\%, India \\ G. D. MUKHERJEE \\ High Pressure Physics Division, Bhabha Atomic Research Centre, \\ Mumbai 400085 , India \\ C. BANSAL \\ School of Physics, University of Hyderabad, Hyderabad 500 046, India \\ ASHOK CHATTERJEE* \\ Department of Physics, Bilkent University, 06800 Bilkent, Ankara, Turkey \\ *ashok@fen.bilkent.edu.tr
}

Received 9 May 2006

\begin{abstract}
Thermal expansion measurements have been performed on a uvarovite rich garnet sample for the first time and compared with the expansion data on grossular and pyrope-rich garnets reported in the literature. A semiclassical model has been used to analyze the data and to obtain various thermodynamic parameters. Using these parameters, the lattice specific heat and the corresponding entropy have also been calculated.
\end{abstract}

Keywords: Uvarovite garnets; thermal expansion.

\section{Introduction}

Garnets are common minerals of metamorphic rocks and the upper mantle peridodites, ${ }^{1}$ and are probably also important phases in the mantle-transition zone (420-670 km) depth. A knowledge of the thermal properties of garnets is therefore of great geophysical importance in the characterization of the thermal structure of the deep interior of the Earth. Considerable effort has been made to study the vibrational and thermodynamic properties of garnets by heat capacity, infra-red, Raman spectroscopic, and ultrasonic techniques and to understand these properties in terms of models for the lattice dynamics of these minerals. ${ }^{2}$ However the same cannot be said about thermal expansion measurements. After the early compilation

*On leave from School of Physics, University of Hyderabad, Hyderabad 500 046, India. 
of thermal expansion data on substances of geological interest, there has been some further work on several minerals, but a full understanding of these measurements is still lacking. ${ }^{3}$ In this work we report the thermal expansion measurements on a uvarovite $\left(\mathrm{Ca}_{3} \mathrm{Cr}_{2} \mathrm{Si}_{3} \mathrm{O}_{12}\right)$ rich garnet sample and compare its behavior with two other members of the garnet family: grossular and pyrope. We show that it is possible to fit the observed data of these minerals using a semi-classical model ${ }^{4}$ and get good estimates of thermodynamic parameters such as the characteristic Debye and Einstein temperatures, Grüneisen parameter, as well as lattice anharmonicity. We also calculate the lattice specific heat and entropy using the parameters obtained from the fits to the thermal expansion data, and compare them with experimentally reported results.

\section{Experiment}

The samples of uvarovite garnets were collected from Bandihalli, Karnataka in India. These garnets occur as euhedral crystals in calc-silicate rocks containing scapolite, plagiodase, quartz and sphene. The calc-silicate rocks form part of a metasedimentary assemblage that consists of quartzite metapetite and banded iron formations. The metapetite consists of cordierite, silliminite, biotate-quartz - feldspar or corderite - anthrophillite - hypersthene garnet - biotate - quartz assemblages. The banded iron formations are composed of garnet - grunarite - magnetite - quartz rich rocks. The composition analysis of the garnet samples by electron microprobe shows that they are uvarovite rich with molar percentages as follows: $72.15 \%$ uvarovite, $11.98 \%$ grossular, $10.11 \%$ andrite, $3.73 \%$ spessartine and $0.66 \%$ pyrope. $^{5}$

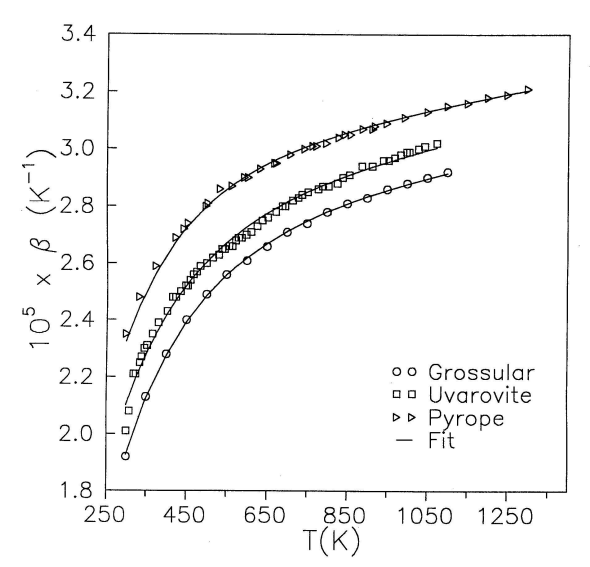

Fig. 1. Variation of thermal expansion coefficient of uvarovite rich garnet as a function of temperature. The expansion behavior of grossular and pyrope rich garnets as reported in the literature are also shown for the sake of comparison. The solid lines through the data points give the fits to our model. 
Thermal expansion measurements were carried out along the longest edge of the sample using a lateral type dilatometer equipped with a sensitive differential transformer. ${ }^{6}$ The system was calibrated against the thermal expansion of corundum. The errors in the thermal expansion coefficient were found to be about 0.5 per cent.

Figure 1 shows the observed coefficient of the volume thermal expansion of the uvarovite rich garnet sample in the temperature range $300 \mathrm{~K}-1073 \mathrm{~K}$, together with the data of Issak et al. ${ }^{7}$ for grossular and that of Suzuki and Anderson ${ }^{8}$ for pyrope rich (72.6 mole\%) garnet. It is clear that the observed thermal expansion coefficient of uvarovite is intermediate between those of grassular and pyrope.

\section{Theoretical Model and Discussion}

The common approach ${ }^{3}$ adopted to interpret thermal expansion data of garnets has been to fit the data to a polynomial expression $\alpha(T)=a_{o}+a_{1} T+a_{2} T^{-2}$ in limited temperature ranges and to quote the values of the constants $a_{o}, a_{1}$ and $a_{2}$. However no physical reason for the origin of the linear and inverse square temperature dependence for $\alpha(T)$ has been provided. In a more recent paper, ${ }^{9}$ the thermal expansion coefficient of 12 garnet end members in the system $(\mathrm{Ca}, \mathrm{Mg}, \mathrm{MnFe})_{3}(\mathrm{Al}, \mathrm{Cr}, \mathrm{Fe})_{2}\left(\mathrm{SiO}_{4}\right)_{3}$ were estimated by Ottonello et al. by using structural simulation and distance least squares refinement techniques wherein the oxygen-cation distances were expressed as empirical functions of mean cation radii and the lattice parameter expressed in terms of the cation-oxygen bond length in the garnet structure. The volume expansion of cation-oxygen polyhedra in the garnet structure were once again assumed to follow the polynomial temperature dependence given earlier by $\mathrm{Fei}^{3}$ and the parameters adjusted by trial and error procedure to match the experimental thermal expansion data. Despite this involved procedure, the agreement between data and calculation can be termed qualitative at best. It was found to be satisfactory for some garnets only over limited temperature ranges. (Fig. 4 of Ref. 9).

As has already been alluded to in the introduction, we have analyzed the thermal expansion behavior of uvarovite garnet using a semiclassical model recently developed by Mukherjee et al. ${ }^{4}$ Its simple minded nature notwithstanding, this approach has been extremely successful in explaining the thermal expansion behavior of various kinds of materials and in predicting their Gruneisen parameters and the characteristic temperatures. ${ }^{4,10-12}$ To introduce this model, we first define the fractional length change of a solid at any temperature $T$ with respect to a reference temperature $T_{o}$ as

$$
\frac{\Delta L}{L\left(T_{o}\right)}=\frac{a(T)-a\left(T_{o}\right)}{a\left(T_{o}\right)}=\frac{\langle x\rangle_{T}-\langle x\rangle_{T_{o}}}{a\left(T_{o}\right)}
$$

where $a(T)$, the interatomic separation at temperature $T$, has been written as $a(T)=a(o)+\langle x\rangle_{T},\langle x\rangle_{T}$ being the average lattice displacement. Since garnet under 
consideration is an insulator, its thermal expansion is essentially due to the anharmonic lattice vibrations which originate from the anharmonic ion-ion interactions. As a result of these interactions, the life-time of a phonon becomes finite and it can decay into other phonons through multi-phonon processes. In this microscopic picture, one usually considers three and four-phonon processes ${ }^{13}$ and calculates the contribution of these processes to the free energy of the harmonic lattice in a perturbative way. Finally the temperature-dependent lattice constant which enters into the theory through the force constant tensor is obtained by minimizing the free energy with respect to it. Though mathematically straightforward and physically elegant, this approach is quite tedious in general and can be intractable at times, depending on the complexity of the system. Furthermore being perturbative in nature, this method may even be inappropriate for systems with strong lattice anharmonicities. Therefore, we have used a rather simplified method in which the effects of the three- and four-phonon interactions are simulated at a semiclassical level by considering an anharmonic potential ${ }^{14}$

$$
V(x)=c x^{2}-g x^{3}-f x^{4}
$$

where $c, g$ and $f$ are constants, $g$ and $f$ measure the strengths of the cubic and quartic anharmonicities respectively. The $x^{3}$-term describes the asymmetry of the mutual ion-ion repulsive potential and the $x^{4}$-term takes care of the flattening of the bottom of the potential well which gives mode softening at large amplitudes. Classically, the average lattice displacement at temperature $T$ for the above potential can be calculated using the Boltzmann distribution. We obtain ${ }^{4}$

$$
\langle x\rangle_{T}=\frac{3 g}{4 c^{2}}\left(k_{B} T\right)\left(1-G\left(k_{B} T\right)-F\left(k_{B} T\right)^{2}\right)
$$

where

$$
G=\frac{15}{16} \frac{g^{2}}{c^{3}}-\frac{8 f}{c^{2}}, \quad F=\frac{35}{16}\left(\frac{15}{4} \frac{g^{2} f}{c^{5}}-\frac{3 f^{2}}{c^{4}}\right)
$$

and terms higher than cubic in $\left(k_{B} T\right)$ have been neglected. In our semiclassical scheme, we replace the classical thermal energy $k_{B} T$ by the average energy of a quantum harmonic oscillator. We use the Debye model for the acoustic phonons and the Einstein approximation for the optical modes. Eq. (3) then reads

$$
\langle x\rangle_{T}=\frac{3 g}{4 c^{2}}\left(\epsilon-G \epsilon^{2}-F \epsilon^{3}\right)
$$

with

$$
\epsilon=\left\{\left(\frac{3}{p}\right) 3 k_{B} T\left(\frac{T}{H_{D}}\right)^{3} \int_{o}^{H_{D / T}} \frac{x^{3} d x}{e^{x}-1}+\frac{(p-3)}{p} \frac{k_{B} \theta_{E}}{e^{\theta_{E / T}}-1}\right\}
$$

where $\theta_{D}$ and $\theta_{E}$ are the Debye and Einstein temperatures respectively and $p$ is the average number of phonon branches excited over the entire range of temperatures. The linear thermal expansion coefficient can now be easily calculated using the 
Table 1. The fitted parameter values and the lattice Grüneisen parameters for grossular, pyrope and uvarovite garnets.

\begin{tabular}{cccc}
\hline Parameters & Grossular & Pyrope & Uvarovite \\
\hline$g^{\prime}\left(e V^{-1}\right)$ & $0.452 \pm 0.025$ & $0.483 \pm 0.075$ & $0.463 \pm 0.050$ \\
$g^{\prime \prime}\left(\mathrm{eV}^{-1}\right)$ & $(14.600 \pm 0.252) \times 10^{-2}$ & $(6.626 \pm 0.747) \times 10^{-2}$ & $(14.557 \pm 0.497) \times 10^{-2}$ \\
$f^{\prime}\left(\mathrm{e} V^{-1}\right)$ & $(0.364 \pm 0.003) \times 10^{-5}$ & $(1.334 \pm 0.075) \times 10^{-5}$ & $(1.305 \pm 0.050) \times 10^{-5}$ \\
$\ominus_{D}(k)$ & $755.776 \pm 1.258$ & $688.403 \pm 3.736$ & $720.550 \pm 2.487$ \\
$p$ & $3.700 \pm 0.252$ & $3.248 \pm 0.747$ & $3.369 \pm 0.497$ \\
$\ominus_{E}(K)$ & $1220.180 \pm 12.585$ & $1241.788 \pm 37.361$ & $1471.967 \pm 24.874$ \\
$\gamma$ & 1.21 & 1.22 & 1.33 \\
\hline
\end{tabular}

equation $\alpha=\frac{1}{a\left(T_{o}\right)} \frac{d\langle x\rangle_{T}}{d T}$. One can also calculate the specific heat (at constant volume) for the anharmonic potential $V(x)$, which is obtained as ${ }^{4}$

$$
C=3 N \epsilon^{\prime}-6 N\left(\frac{15 g^{2}}{8 c^{3}}-\frac{3 f}{4 c^{2}}\right) \epsilon \epsilon^{\prime}
$$

where

$$
\begin{aligned}
\epsilon^{\prime}= & \left\{\left(\frac{3}{p}\right) 3 k_{B}\left(\frac{T}{\theta_{D}}\right)^{3} \int_{o}^{\theta_{D / T}} \frac{z^{4} e^{z}}{\left(e^{z}-1\right)^{2}} d z\right. \\
& \left.+\left(\frac{p-3}{p}\right) k_{B}\left(\frac{\theta_{E}}{T}\right)^{2} \frac{e^{\theta_{E / T}}}{\left(e^{\theta_{E / T}}-1\right)^{2}}\right\}
\end{aligned}
$$

and $N$ is the total number of atoms in the crystal. The calculation of (vibrational) entropy is now straightforward. One can also obtain the (vibrational) Grüneisen parameter using the well-known relation $\gamma=\frac{\beta B_{T} V}{C}$, where $\beta$ is the coefficient of volume expansion and can be taken to be equal to $3 \alpha$ for isotropic systems, $B_{T}$ is the isothermal bulk modulus and $V$ is the volume of the system.

Figure 1 shows the fits to the volume expansion coefficient data of uvarovite garnet obtained by us along with the fits to the earlier data on grossular ${ }^{7}$ and natural pyrope. ${ }^{4}$ It is evident that the fits are excellent. The parameters obtained from the best fit are shown in Table 1.

It is again clear from the values of the anharmonicity parameters that in view of the lattice anharmonicity, uvarovite lies somewhere in between grossular and pyrope. Using these parameters, we calculate the specific heat and entropy of all the three garnets. It is clear that for grossular, the results predicted by our model are in excellent agreement with the reported data (Fig. 2) and for pyrope, the agreement is also fairly good (Fig. 3). This imparts a fair amount of confidence in our model. The slight discrepancy in the case of pyrope is probably due to the substantial presence of other components in this garnet.

In Fig. 4 we have shown the specific heat and entropy behavior of uvarovite rich garnet as predicted from our model. We could not however make any comparison 


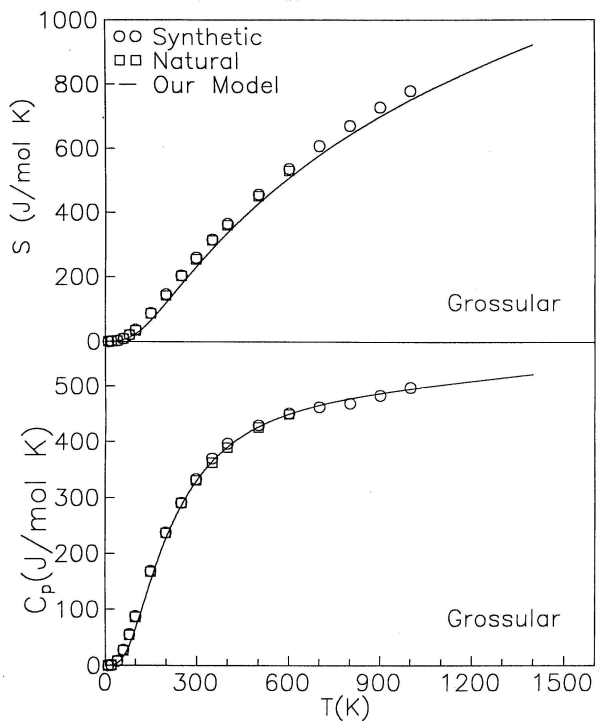

Fig. 2. Temperature variation of lattice specific heat $\left(C_{p}\right)$ and entropy $(S)$ of grossular as calculated from our model together with the reported data of Isaak et al. ${ }^{7}$

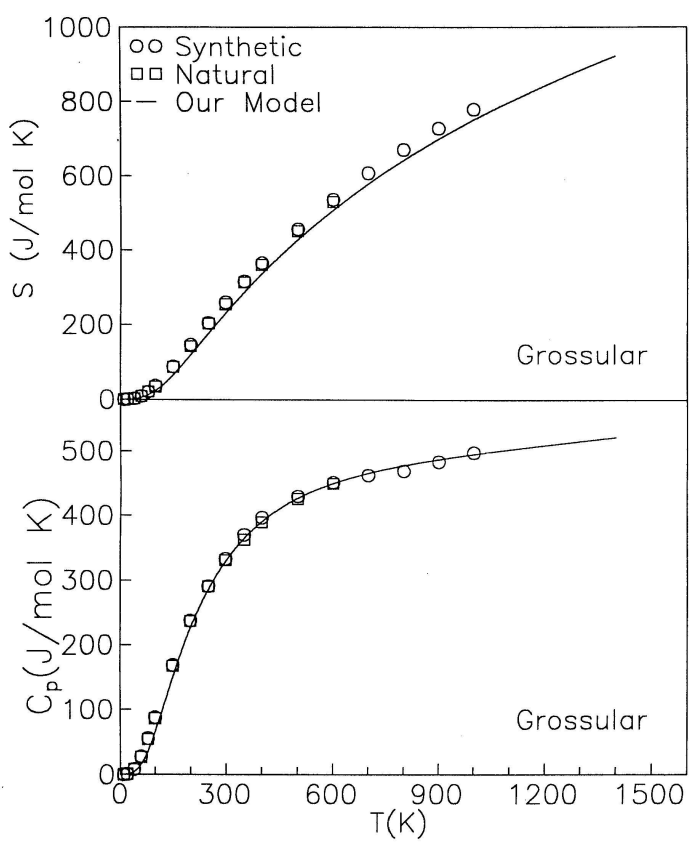

Fig. 3. Temperature variation of lattice specific heat $\left(C_{p}\right)$ and entropy $(S)$ of a pyrope rich garnet as calculated from our model, together with the reported data of Suzuki and Anderson. ${ }^{8}$ 


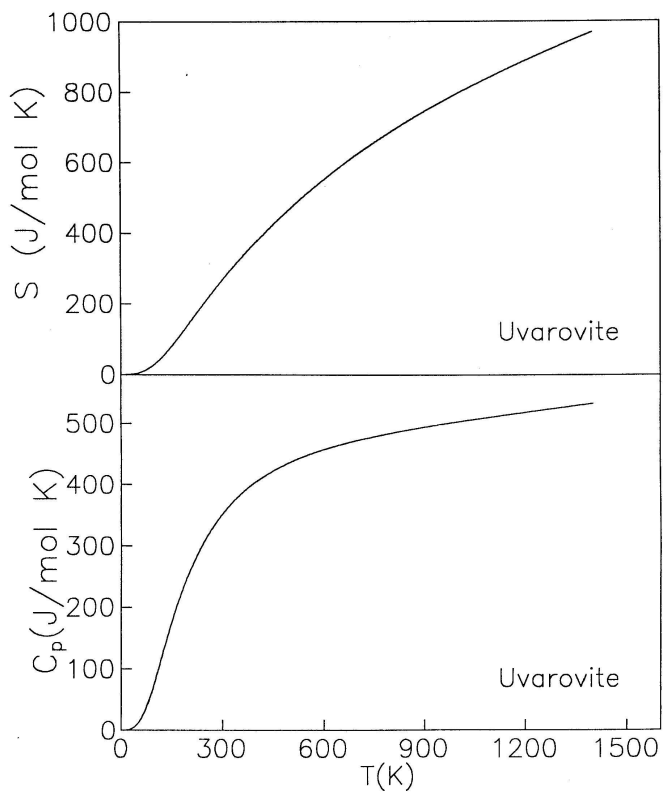

Fig. 4. Temperature variation of lattice specific heat $\left(C_{p}\right)$ and entropy $(S)$ of a uvarovite rich garnet as calculated from our model.

with these results, for no experimental data, to our knowledge, on the specific heat of uvarovite are available in the literature.

\section{Conclusion}

In conclusion, we have performed thermal expansion measurements on a uvarovite rich garnet sample and compared its behavior with that of grossular and pyrope. We have analyzed the expansion data of these garnets using a recently developed semiclassical model to obtain various thermodynamic parameters such as lattice anharmonicity parameters, Grüneisen constant and the characteristic Debye and Einstein temperatures. We have also calculated the lattice specific heat and entropy using the parameters obtained from our fits to the thermal expansion data. Comparison of these results with the available experimental data shows that our model provides results which are in good agreement with the experimental values.

\section{References}

1. T. S. Duffy and D. L. Andersaon, J. Geophys. Res. 94, 1895 (1989).

2. S. W. Kieffer, Reviews of Geophysics and Space Physics 17, 1 (1979).

3. Y. Fei, A Handbook of Physical Constants (AGO Reference Shelf 2, 1995), p. 29.

4. G. D. Mukherjee, C. Bansal and A. Chatterjee, Phys. Rev. Lett. 76, 1876 (1996).

5. G. Parthasarathy, V. Balaram and R. Srinivasan, J. Asian Earth Sciences 17, 345 (1999). 
6. Y. V. Ramana and L. P. Sarma, Phys. Earth. Planet. Inter. 22, 36 (1980).

7. D. G. Isaak, O. L. Anderson and O. Hitoshi, Phys. Chem. Minerals 19, 106 (1992).

8. I. Suzuki and O. L. Anderson, J. Phys. Earth 31, 125 (1983).

9. G. Ottonello, M. Bokreta and P. F. Sciuto, American Mineralogist 81, 429 (1996).

10. G. D. Mukherjee, C. Bansal and A. Chatterjee, Solid State Communications 104, 657 (1997).

11. G. D. Mukherjee, C. Bansal and A. Chatterjee, Int. J. Mod. Phys. B 12, 449 (1998).

12. G. D. Mukherjee, A. Chatterjee and C. Bansal, Physica C 232, 241 (1994).

13. O. Madelung, Introduction to Solid State Theory (Springer, Berlin, 1978).

14. C. Kittel, Introduction to Solid State Physics (Wiley Eastern Ltd., New Delhi, 1977), p. 142 . 